\title{
Ambient pressure synthesis of corundum-type
}

\author{
$\mathrm{In}_{2} \mathrm{O}_{3}$
}

Mauro Epifani, Pietro Siciliano

Istituto per la Microelettronica e i Microsistemi, IMM-CNR, Sezione di Lecce, Via Arnesano, 73100 Lecce, Italy

Alexander Gurlo*, Nicolae Barsan, Udo Weimar

Institute of Physical and Theoretical Chemistry, University of Tübingen, Auf der Morgenstelle 8, 72076 Tübingen, Germany

Supporting information

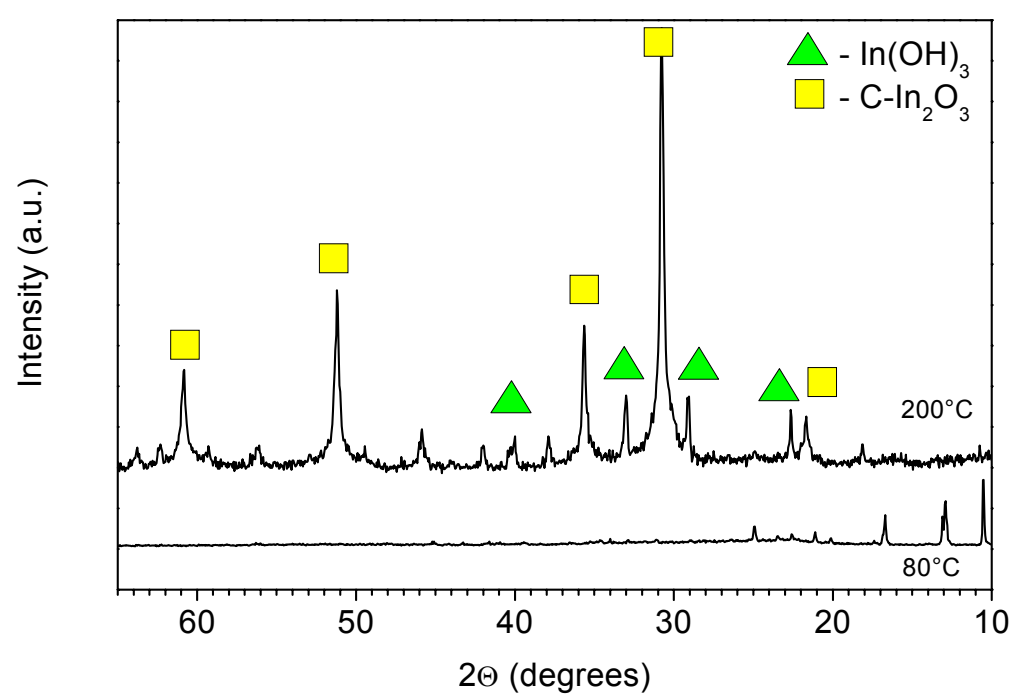

Figure 1. X-ray powder diffraction pattern recorded from the powders prepared in acetylacetone-methanol-based route. 


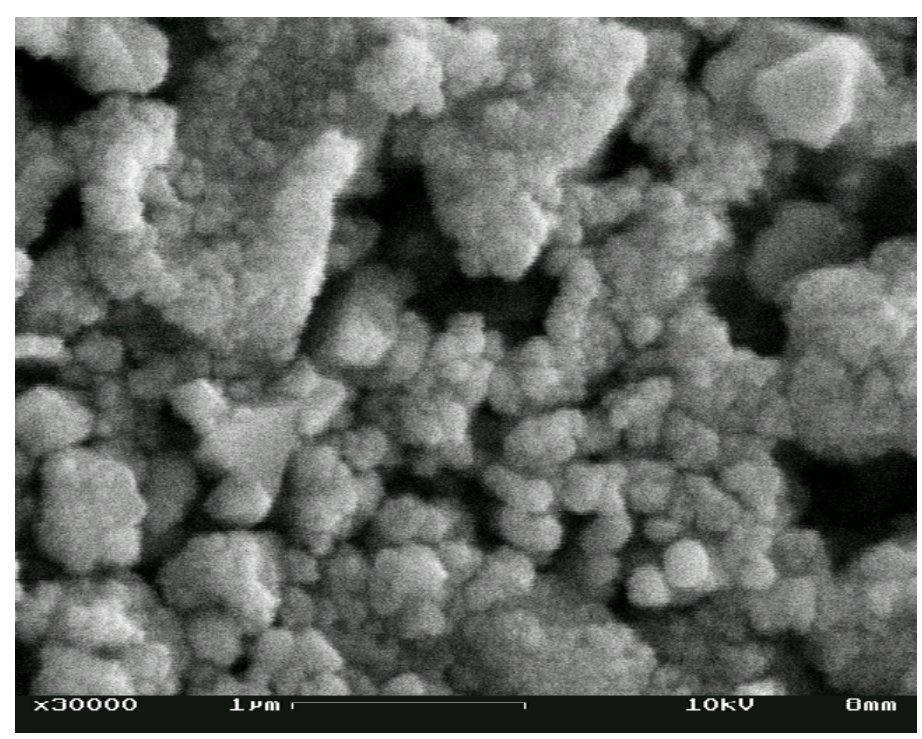

Figure 2. SEM of the powder prepared in acetylacetone-methanol-based route. Calcination temperature: $500^{\circ} \mathrm{C}$. 